\title{
Faune ichtyologique du lac de barrage Solomougou (Korhogo, Côte d'Ivoire)
}

\author{
Koffi Dongo KOUASSI ${ }^{1 *}$, Moustapha DIABY ${ }^{2 *}$, Yaya SORO $^{3}$ et Konan N'DA ${ }^{3}$ \\ ${ }^{1}$ UFR des Sciences Biologiques, Université Peleforo Gon Coulibaly, Korhogo, Côte d'Ivoire. \\ ${ }^{2}$ Institut de Gestion Agropastorale, Université Peleforo Gon Coulibaly, Korhogo, Côte d'Ivoire. \\ ${ }^{3} U F R$ des Sciences de la Nature, Université Nangui Abrogoua, Abidjan, Côte d'Ivoire. \\ *Auteur correspondant, E-mail: dongokoff2003@gmail.com, Tél : +22508072509
}

\section{RÉSUMÉ}

Le lac de barrage Solomougou, créé en 1973 à des fins agricoles, est exploité de nos jours pour les activités halieutiques. La présente étude y a été conduite en vue d'étudier sa diversité ichtyologique. Les captures des pêcheurs ont été analysées de janvier à décembre 2019. Les paramètres étudiés sont la richesse spécifique, les abondances spécifique et relative, le pourcentage d'occurrence ainsi que les indices de diversité spécifique de Shannon et Weaver et d'équitabilité de Piélou. Ainsi, 12950 spécimens répartis en quatorze familles, vingt-cinq genres et trente-sept espèces ont été répertoriés. Les trois familles comptant le plus d'espèces sont les Cichlidae (10 espèces), suivis des Mormyridae ( 6 espèces) et des Cyprinidae ( 5 espèces). Cependant, dans les captures totales, les Cichlidae $(41,87 \%)$ sont toujours les plus nombreux, suivis des Alestidae $(14,47 \%)$ et des Mochokidae $(12,84 \%)$, chacune de ces deux dernières familles étant représentées par deux espèces. Enfin, en termes d'abondance relative spécifique, Brycinus imberi $(14,41 \%)$, Synodontis schall $(12,76 \%)$ et Oreochromis niloticus $(11,62 \%)$ sont les plus représentatives. Relativement aux valeurs de fréquence d'occurrence, la pêcherie compte quatorze espèces accidentelles, une accessoire et vingtdeux constantes. Les valeurs de l'indice de diversité spécifique de Shannon et de l'indice d'équitabilité sont respectivement 3,9159 et 0,7517.

(c) 2020 International Formulae Group. All rights reserved.

Mots clés : Espèces de poissons, abondance, distribution, lac de barrage Solomougou, Côte d'Ivoire.

\section{Ichtyological fauna of the Solomougou dam lake (Korhogo, Côte d'Ivoire)} \begin{abstract}
The Solomougou dam lake, created in 1973 for crop irrigation, is nowadays used for fishing activities. This study was conducted in order to understand the ichthyological diversity in this fishery. So, fishermen's catches have been analyzed from January to December 2019 in order to identify fish species and to calculate their specific and relative abundances, their occurrence's frequency as well as Shannon diversity index and Piélou equitability index. Thus, 12950 specimens including fourteen families, twenty-five genera and thirty-seven species have been recorded. The three families with the most species are Cichlidae (10 species), followed by
\end{abstract}


Mormyridae (6 species) and Cyprinidae (5 species). However, taking into account the relative abundances of families in the total catches, Cichlidae (41.87\%) are always the most numerous, followed by Alestidae (14.47\%) and Mochokidae (12.84\%), each of these last two families being represented by only two species. Finally, in terms of specific relative abundance, the three most dominant species are Brycinus imberi (14.41\%), Synodontis schall $(12.76 \%)$ and Oreochromis niloticus $(11.62 \%)$. With regard to the occurrence's frequency values, fourteen accidental species, one accessory and twenty-two constants have been identified. Shannon diversity index and Piélou equitability index are 3.9159 and 0.7517 , respectively.

(C) 2020 International Formulae Group. All rights reserved.

Keywords: Fish species, abundance, distribution, Solomougou Dam Lake, Côte d'Ivoire.

\section{INTRODUCTION}

Les écosystèmes aquatiques abritent une grande diversité biologique. L'étude de cette diversité biologique est importante pour comprendre le fonctionnement de ces milieux, par exemple la survie des différentes espèces, lesquelles dépendent les unes des autres (Liquete et al., 2016). Les ressources ichtyologiques représentent les principales sources de protéines animales accessibles pour beaucoup de populations. En 2017 par exemple, à l'échelle mondiale, la consommation moyenne de poisson, estimée par personne, était de l'ordre de 20,5 kg (FAO, 2018).

En Côte d'Ivoire, les lacs de barrage du nord, construits depuis les années 1970 et dont le nombre est estimé à 210 (Le Guen, 2002), étaient initialement utilisés à des fins agricole et pastorale (Cecchi et al., 2007). Cependant, de nos jours, ils représentent un réel potentiel halieutique. Les études sur la pêche y ont particulièrement été conduites sur ceux de petites et moyennes superficies. Elles ont porté entre autres sur les aspects économiques de l'exploitation de leurs ressources halieutiques (Koffi, 2000), le potentiel halieutique de ces pêcheries (Da Costa et al., 2004) ou encore les structures trophiques de leurs peuplements (Da Costa et Tito de Morais, 2007). En revanche, les plus grandes retenues ont fait l'objet de peu d'études. C'est le cas pour le lac de barrage Solomougou d'une superficie de 500hectares. Cet hydrosystème, le plus grand à proximité de la plus grande localité du nord du pays (la ville de Korhogo), est exploité pour la pêche durant toutes les périodes de l'année et dont les captures approvisionnent le marché local. Le présent travail a donc été conduit dans cette pêcherie afin d'étudier la composition ichtyologique des captures.

\section{MATÉRIEL ET MÉTHODES Milieu d'étude}

Cette étude a été conduite sur le lac de barrage Solomougou. Cet hydrosystème a été aménagé depuis 1973 sur la rivière Solomougou, un affluent du fleuve Bandama, et est localisé au nord de la Côte d'Ivoire. Son bassin versant se situe entre les méridiens $5^{\circ} 30$ 'et $6^{\circ}$ Ouest et les parallèles $9^{\circ}$ et $9^{\circ} 30^{\prime}$ Nord (ORSTOM, 1962). Il couvre 250 kilomètres carrés de bassin versant et une superficie maximale de plan d'eau de 500 hectares (Traoré, 1996). Le climat de la zone est caractérisé par deux saisons. La saison sèche couvre la période de novembre à avril et celle des pluies de mai à octobre. Les précipitations annuelles moyennes restent inférieures à 1200 $\mathrm{mm}$ (Brou, 2005). Les températures moyennes journalières s'élèvent à $29^{\circ} \mathrm{C}$ environ en saison sèche et à $25^{\circ} \mathrm{C}$ en saison des pluies (Yapo et al., 2016).

\section{Conduite de l'échantillonnage}

Les échantillons utilisés dans le cadre de la présente étude proviennent des captures de l'ensemble des pêcheurs fréquentant le lac de barrage. Ces captures ont été effectuées les dix premiers jours de chaque mois de janvier à décembre 2019. Différents types d'engins de pêche étaient utilisés par les pêcheurs. Ce sont 
les nasses, les filets maillants, les filets éperviers, les palangres, les pièges en bambou et les sennes. Cependant, les espèces capturées n'étaient pas reparties par type d'engin de pêche. Tous les spécimens capturés par un pêcheur donné étaient identifiés in situ dans la mesure du possible. Dans le cas contraire, des échantillons comptant la moitié ou le tiers des captures étaient prélevés de façon aléatoire pour identification. La taille de ces échantillons était souvent limitée par l'impatience des pêcheurs à convoyer leurs captures sur le marché pour la vente. En tout état de cause, l'objectif était d'analyser la composition des captures de chaque pêcheur. Les poissons ont été identifiés selon le guide défini par Stiassny et al. (2008).

\section{Analyse des données}

Les indices utilisés pour évaluer le peuplement ichtyologique sont la richesse spécifique (S), les abondances spécifique et relative, le pourcentage d'occurrence $(\mathrm{F})$ ainsi que les indices de diversité spécifique (H') de Shannon et Weaver (1963) et d'équitabilité (E) de Piélou (1966).

a- La richesse spécifique (S) exprime le nombre d'espèces rencontrées dans les captures.

b- L'abondance spécifique correspond au nombre de spécimens d'une espèce dans les captures.

c- L'abondance relative exprime le rapport en pourcentage de l'effectif d'une espèce donnée sur l'effectif total des poissons capturés.

d- Le pourcentage d'occurrence (F) est le rapport exprimé en pourcentage du nombre de prélèvements dans lesquels se trouve une espèce donnée $(\mathrm{Fi})$ sur le nombre total des prélèvements $(\mathrm{Ft}): \mathrm{F}=(\mathrm{Fi} * 100) / \mathrm{Ft}$. Selon la valeur de $\mathrm{F}$, l'espèce est dite constante si $\mathrm{F} \geq 50 \%$, accessoire lorsque $25 \% \leq \mathrm{F} \leq 50$ $\%$ et accidentelle lorsque $\mathrm{F}<25 \%$ (Dajoz, 2000). e- L'indice de diversité (H') de Shannon et Weaver (1963) mesure la diversité spécifique à partir de la formule : $\mathrm{H}^{\prime}=$ $-\sum_{i=1}^{S} \frac{n i}{N} X \log 2 \frac{n i}{N}$ avec :

$\mathrm{S}$ : nombre total d'espèces présentes ; ni : effectif de l'espèce i dans l'échantillon ; $\mathrm{N}$ : effectif total. $H^{\prime}$ ' varie entre 0 , dans le cas où le peuplement n'est constitué que d'une seule espèce, et $\log 2 \mathrm{~S}$ dans le cas où toutes les espèces présentes le sont avec une abondance équivalente ( $H^{\prime}=4,5$ ou 5 pour les peuplements les plus diversifiés).

f- $\quad$ L'indice d'équitabilité (E) de Pielou (1966) permet d'apprécier la qualité de la répartition des individus au sein des espèces du milieu. Il est calculé à l'aide de la formule : E $=\mathrm{H}^{\prime} / \log 2 \mathrm{Rs}, \mathrm{H}^{\prime}$ correspondant à l'indice de diversité (H') de Shannon et Weaver et Rs au nombre d'espèces. La valeur de $\mathrm{E}$ est comprise entre 0 et 1 . Elle tend vers 0 quand la quasitotalité des effectifs des individus est concentrée sur une espèce, et vers 1 lorsque toutes les espèces ont la même abondance (état d'équilibre).

\section{RÉSULTATS}

\section{Composition et abondances relatives des espèces}

$\mathrm{Au}$ terme de cette étude, 12950 spécimens répartis en quatorze (14) familles, vingt-cinq (25) genres et trente-sept (37) espèces ont été répertoriés. En termes de diversité au sein des familles, les plus représentées sont les Cichlidae qui comptent dix espèces, suivis des Mormyridae et des Cyprinidae avec des effectifs respectifs de six et cinq espèces. Les autres familles sont représentées par une ou deux espèces.

Cependant, en considérant les abondances relatives des familles dans les captures totales, les Cichlidae $(41,87 \%)$ sont toujours les plus nombreux, en rapport avec leur richesse spécifique, suivis des Alestidae $(14,47 \%)$ et des Mochokidae (12,84\%) qui, cependant, ne sont représentées chacune que 
seulement par deux espèces. Les Mormyridae et Cyprinidae, qui comptent respectivement six et cinq espèces, ne représentent cependant que $8,78 \%$ et $2,97 \%$ des captures.

Enfin, concernant l'abondance relative des espèces, les trois espèces les plus représentatives dans des proportions variant entre 10 et $15 \%$ sont Brycinus imberi $(14,41 \%)$, Synodontis schall $(12,76 \%)$ et Oreochromis niloticus $(11,62 \%)$. Elles sont suivies de Sarotherodon galilaeus (8,96\%), Tilapia mariae $(8,61 \%)$, Clarias gariepinus $(8,00 \%)$ et Hemichromis fasciatus (7,00\%). Toutes les autres espèces sont moins représentées, dixhuit (18) d'entre elles ayant chacune une proportion inférieure à $1 \%$ (Tableau 1).

\section{Distribution temporelle}

Les proportions relatives des différentes espèces, relevées dans les captures totales pendant l'échantillonnage, ont permis d'obtenir le Tableau 2. Les résultats indiquent une grande variabilité de leurs pics d'abondance tout le long de l'année. Les fréquences d'occurrence ont permis de recenser quatorze espèces accidentelles, une accessoire et vingt-deux constantes.

Le premier groupe est représenté par Barbus sacratus, Barbus wurtzi, Brycinus nurse, Hemichromis bimaculatus, Malopterurus electricus,Marcusenius senegalensis, Papyrocranus afer, Pollimyrus isidori, Polypterus endlicheri, Protopterus annectens, Sarotherodon melanotheron, Synodontis nigrita, Tilapia busumana et Tilapia guineensis. Les périodes d'apparition de ces espèces sont variables et limitées entre un (01) et trois (3) mois au cours de l'année.

Quant au second groupe, il compte une seule espèce (Schilbe mystus) avec une fréquence d'occurrence de $33.33 \%$.

Le dernier groupe, représentant les espèces dites constantes, en compte vingt-deux (22). Cependant, dans ce groupe, certaines espèces ont été rencontrées dans les captures durant tous les mois de l'année. Ce sont Brycinus imberi, Chrisychthys nigrodigitatus, Chromidotilapia guntheri, Clarias gariepinus, Hemichromis fasciatus, Labeo coubie, Labeo parvus, Marcusenius furcidens, Marcusenlus ussheri, Oreochromis niloticus, Petrocephalus Bovei, Sarotherodon galilaeus, Schilbe intermedius, Synodontis schall et Tilapia mariae. Les autres représentants de ce groupe apparaissent entre 7 et 11 mois dans les captures au cours de l'année. Ce sont Chrysichthys maurus, Ctenopoma patherici, Heterobranchus longifilis, Heterotis niloticus, Mormyrus rume, Raiamas senegalensis et Tilapia zillii.

\section{Indices écologiques}

Les valeurs de l'indice de diversité spécifique (H') de Shannon et Weaver et de l'indice d'équitabilité (E) de Piélou sont respectivement de 3,9159 et de 0,7517 .

Tableau 1: Composition et abondances relatives des familles et espèces de poissons.

\begin{tabular}{|c|c|c|c|c|}
\hline \multirow[b]{2}{*}{ Familles } & \multirow[b]{2}{*}{ Espèces } & \multirow[b]{2}{*}{ Effectifs } & \multicolumn{2}{|c|}{ Abondances relatives (\%) } \\
\hline & & & Par espèce & Par famille \\
\hline & Brycinus imberi & 1866 & 14,41 & \\
\hline Alestidae & Brycinus nurse & 8 & 0,06 & 14,47 \\
\hline Anabantidae & Ctenopoma patherici & 27 & 0,21 & 0,21 \\
\hline & Chromidotilapia guntheri & 248 & 1,92 & \\
\hline & Hemichromis bimaculatus & 6 & 0,05 & \\
\hline
\end{tabular}




\begin{tabular}{|c|c|c|c|c|}
\hline & Hemichromis fasciatus & 906 & 7,00 & \\
\hline & Oreochromis niloticus & 1505 & 11,62 & \\
\hline \multirow[t]{7}{*}{ Cichlidae } & Sarotherodon galilaeus & 1160 & 8,96 & 41,87 \\
\hline & Sarotherodon melanotheron & 18 & 0,14 & \\
\hline & Tilapia busumana & 2 & 0,02 & \\
\hline & Tilapia guineensis & 21 & 0,16 & \\
\hline & Tilapia mariae & 1115 & 8,61 & \\
\hline & Tilapia zillii & 441 & 3,41 & \\
\hline & Clarias gariepinus & 1036 & 8,00 & \\
\hline \multirow[t]{2}{*}{ Clariidae } & Heterobranchus longifilis & 129 & 1,00 & 9,00 \\
\hline & Chrysichthys maurus & 252 & 1,95 & \\
\hline \multirow[t]{3}{*}{ Claroteidae } & Chrisychthys nigrodigitatus & 529 & 4,08 & 6,03 \\
\hline & Barbus sacratus & 8 & 0,06 & \\
\hline & Barbus wurtzi & 7 & 0,05 & \\
\hline \multirow[t]{3}{*}{ Cyprinidae } & Labeo coubie & 213 & 1,64 & 2,97 \\
\hline & Labeo parvus & 138 & 1,07 & \\
\hline & Raiamas senegalensis & 18 & 0,14 & \\
\hline \multirow[t]{2}{*}{ Malapteruridae } & Malopterurus electricus & 4 & 0,03 & 0,03 \\
\hline & Synodontis nigrita & 10 & 0,08 & \\
\hline \multirow[t]{3}{*}{ Mochokidae } & Synodontis schall & 1653 & 12,76 & 12,84 \\
\hline & Marcusenius furcidens & 322 & 2,49 & \\
\hline & Marcusenius senegalensis & 1 & 0,01 & \\
\hline \multirow[t]{4}{*}{ Mormyridae } & Marcusenlus ussheri & 253 & 1,95 & 8,78 \\
\hline & Mormyrus rume & 314 & 2,42 & \\
\hline & Petrocephalus Bovei & 245 & 1,89 & \\
\hline & Pollimyrus isidori & 2 & 0,02 & \\
\hline Notopteridae & Papyrocranus afer & 7 & 0,05 & 0,05 \\
\hline Osteoglossidae & Heterotis niloticus & 29 & 0,22 & 0,22 \\
\hline Polypteridae & Polypterus endlicheri & 5 & 0,04 & 0,04 \\
\hline \multirow[t]{2}{*}{ Protopteridae } & Protopterus annectens & 5 & 0,04 & 0,04 \\
\hline & Schilbe intermedius & 439 & 3,39 & \\
\hline \multirow[t]{2}{*}{ Schilbeidae } & Schilbe mystus & 8 & 0,06 & 3,45 \\
\hline & Total & 12950 & 100,00 & 100,00 \\
\hline
\end{tabular}


K. D. KOUASSI et al. / Int. J. Biol. Chem. Sci. 14(7): 2528-2537, 2020

Tableau 2: Variations mensuelles (de janvier à décembre) des abondances relatives et pourcentages d'occurrence (F) des différentes espèces dans les captures.

\begin{tabular}{|c|c|c|c|c|c|c|c|c|c|c|c|c|c|c|}
\hline Espèces & $\mathbf{J}$ & F. & M. & A. & M. & J. & $\mathbf{J}$. & A. & S. & O. & N. & D. & $\mathbf{F}$ & Effectifs \\
\hline Barbus sacratus & & 100,00 & & & & & & & & & & & 8,33 & 8 \\
\hline Barbus wurtzi & & 100,00 & & & & & & & & & & & 8,33 & 7 \\
\hline Brycinus imberi & 3,64 & 9,22 & 11,74 & 8,68 & 13,13 & 6,86 & 5,41 & 3,27 & 3,75 & 3,64 & 18,81 & 11,85 & 100,00 & 1866 \\
\hline Brycinus nurse & & 25,00 & 75,00 & & & & & & & & & & 16,67 & 8 \\
\hline Chrysichthys maurus & & 13,89 & 19,05 & 24,21 & 15,48 & 8,73 & 8,73 & 3,17 & 3,57 & 3,17 & & & 75,00 & 252 \\
\hline $\begin{array}{c}\text { Chrisychthys } \\
\text { nigrodigitatus }\end{array}$ & 9,07 & 5,86 & 6,43 & 2,46 & 2,08 & 5,67 & 5,48 & 17,01 & 8,32 & 5,86 & 10,59 & 21,17 & 100,00 & 529 \\
\hline $\begin{array}{c}\text { Chromidotilapia } \\
\text { guntheri }\end{array}$ & 23,39 & 14,11 & 11,29 & 5,24 & 4,84 & 3,63 & 20,16 & 3,63 & 2,42 & 2,02 & 2,82 & 6,45 & 100,00 & 248 \\
\hline Clarias gariepinus & 7,72 & 6,95 & 7,92 & 8,69 & 6,37 & 7,14 & 8,40 & 12,36 & 8,40 & 7,43 & 9,27 & 9,36 & 100,00 & 1036 \\
\hline Ctenopoma patherici & 7,41 & 25,95 & 33,33 & 3,70 & 3,70 & & 3,70 & 3,70 & & & 3,70 & 14,81 & 75,00 & 27 \\
\hline $\begin{array}{c}\text { Hemichromis } \\
\text { bimaculatus }\end{array}$ & 50,00 & 50,00 & & & & & & & & & & & 16,67 & 6 \\
\hline $\begin{array}{c}\text { Hemichromis } \\
\text { fasciatus }\end{array}$ & 10,60 & 5,41 & 7,84 & 6,84 & 4,08 & 2,98 & 5,19 & 5,19 & 6,40 & 6,84 & 16,23 & 22,41 & 100,00 & 906 \\
\hline $\begin{array}{c}\text { Heterobranchus } \\
\text { longifilis }\end{array}$ & & 23,26 & 3,88 & 0,78 & & 1,55 & 3,10 & 27,13 & 23,26 & $\begin{array}{c}14,7 \\
3\end{array}$ & 2,33 & & 75,00 & 129 \\
\hline Heterotis niloticus & 3,45 & 17,24 & 37,93 & 10,34 & 3,45 & & & & & & 3,45 & 24,14 & 58,33 & 29 \\
\hline Labeo coubie & 1,88 & 26,29 & 24,41 & 15,02 & 9,86 & 1,88 & 0,94 & 0,94 & 0,47 & 0,94 & 3,76 & 13,62 & 100,00 & 213 \\
\hline Labeo parvus & 7,97 & 21,01 & 15,22 & 9,42 & 3,62 & 1,45 & 5,80 & 5,80 & 4,35 & 5,07 & 5,07 & 15,22 & 100,00 & 138 \\
\hline $\begin{array}{c}\text { Malopterurus } \\
\text { electricus }\end{array}$ & & 25,00 & 25,00 & & & & 50,00 & & & & & & 25,00 & 4 \\
\hline $\begin{array}{l}\text { Marcusenius } \\
\text { furcidens }\end{array}$ & 25,16 & 18,32 & 13,35 & 2,80 & 1,24 & 6,83 & 3,11 & 1,24 & 3,42 & 3,42 & 14,60 & 6,52 & 100,00 & 322 \\
\hline
\end{tabular}


K. D. KOUASSI et al. / Int. J. Biol. Chem. Sci. 14(7): 2528-2537, 2020

\begin{tabular}{|c|c|c|c|c|c|c|c|c|c|c|c|c|c|c|}
\hline \multirow[b]{2}{*}{$\begin{array}{l}\text { Marcusenius } \\
\text { senegalensis }\end{array}$} & \multirow{2}{*}{\multicolumn{2}{|c|}{100,00}} & & \multirow[b]{2}{*}{$8,33,00$} & \multirow[b]{2}{*}{1} \\
\hline & & & & & & & & & & & & & & \\
\hline Marcusenlus ussheri & 30,04 & 12,25 & 18,97 & 4,35 & 4,35 & 7,91 & 7,91 & 2,77 & 1,19 & 1,19 & 1,98 & 7,11 & 100,00 & 253 \\
\hline Mormyrus rume & 8,28 & 26,75 & 1,27 & 3,18 & 1,59 & 2,87 & & 0,32 & 0,64 & 2,55 & 28,98 & 23,57 & 91,67 & 314 \\
\hline $\begin{array}{c}\text { Oreochromis } \\
\text { niloticus }\end{array}$ & 5,25 & 4,25 & 5,32 & 13,69 & 13,95 & 25,85 & 18,67 & 3,65 & 3,19 & 1,73 & 2,19 & 2,26 & 100,00 & 1505 \\
\hline Papyrocranus afer & & & 100,00 & & & & & & & & & & 8,33 & 7 \\
\hline Petrocephalus Bovei & 1,22 & 13,06 & 1,22 & 1,22 & 13,06 & 8,57 & 46,12 & 1,22 & 2,04 & 3,67 & 5,31 & 3,27 & 100,00 & 245 \\
\hline Pollimyrus isidori & & 100,00 & & & & & & & & & & & 8,33 & 2 \\
\hline Polypterus endlicheri & & & 80,00 & & & 20,00 & & & & & & & 16,67 & 5 \\
\hline $\begin{array}{c}\text { Protopterus } \\
\text { annectens }\end{array}$ & & & & 100,00 & & & & & & & & & 8,33 & 5 \\
\hline Raiamas senegalensis & 22,22 & & 16,67 & 5,56 & 11,11 & 11,11 & & 5,56 & & & 16,67 & 11,11 & 66,67 & 18 \\
\hline $\begin{array}{c}\text { Sarotherodon } \\
\text { galilaeus }\end{array}$ & 5,00 & 8,53 & 9,74 & 10,69 & 11,98 & 10,00 & 5,95 & 3,19 & 1,81 & 2,16 & 16,64 & 14,31 & 100,00 & 1160 \\
\hline $\begin{array}{l}\text { Sarotherodon } \\
\text { melanotheron }\end{array}$ & & 38,89 & 50 & & 11,11 & & & & & & & & 25,00 & 18 \\
\hline Schilbe intermedius & 27,33 & 14,12 & 7,29 & 0,68 & 4,78 & 0,91 & 2,28 & 0,46 & 3,42 & 4,1 & 10,25 & 24,37 & 100,00 & 439 \\
\hline Schilbe mystus & & 50 & 12,5 & & & 25 & & 12,5 & & & & & 33,33 & 8 \\
\hline Synodontis nigrita & & & 40 & & 20 & & & & & & 40 & & 25,00 & 10 \\
\hline Synodontis schall & 6,96 & 6,41 & 14,16 & 7,68 & 6,41 & 4,05 & 4,84 & 10,47 & 8,89 & 7,8 & 11,62 & 10,71 & 100,00 & 1653 \\
\hline Tilapia busumana & & 100 & & & & & & & & & & & 8,33 & 2 \\
\hline Tilapia guineensis & 80,95 & 4,76 & 14,29 & & & & & & & & & & 25,00 & 21 \\
\hline Tilapia mariae & 13,18 & 6,91 & 3,05 & 4,04 & 6,46 & 4,04 & 8,7 & 10,67 & 5,38 & 5,11 & 12,02 & 20,45 & 100,00 & 1115 \\
\hline Tilapia zillii & & 21,77 & 42,4 & 11,79 & 2,95 & 2,95 & 13,15 & 2,72 & 1,36 & 0,91 & & & 75,00 & 441 \\
\hline
\end{tabular}




\section{DISCUSSION}

Les lacs de barrage du nord de la Côte d'Ivoire sont caractérisés par une variation de leur composition ichtyologique d'une retenue à une autre. A titre d'exemple, DA Costa et Tito De Morais (2007), dans leurs travaux dans seize lacs de barrage dans cette région du pays, ont relevé une variation du nombre d'espèces par retenue de 18 à 37. Ces auteurs ont également noté qu'il n'y a pas de relation significative entre la richesse spécifique et la taille des réservoirs.

L'effectif de trente-sept (37) espèces de poissons inventoriées dans le lac de barrage Solomougou se trouve donc comparable au maximum enregistré par ces auteurs.

En outre, un inventaire du peuplement ichtyologique du lac de barrage Solomougou depuis plus de deux décennies faisait état de la présence de 33 espèces (Traoré, 1996). Cependant, douze espèces répertoriées dans le rapport de cet auteur n'ont pas été rencontrées dans la présente étude. Ce sont: Alestes baremoze, Brycinus longipinnis, Mormyrops anguilloides, Barbus trispilos, Barbus ablabes, Barbus macrops, Labeo senegalensis, Hepsetus odoe, Clarias anguillaris, Schilbe mandibularis, Auchenoglanis occidentalis et Ctenopomakings leyae. A l'opposé, quinze nouvelles espèces ont été signalées dans le cadre de ce travail. Ce sont: Ctenopoma patherici, Sarotherodon melanotheron, Tilapia busumana, Tilapia guineensis, Tilapia mariae, Barbus sacratus, Barbus wurtzi, Malopterurus electricus, Synodontis nigrita, Marcusenius senegalensis, Pollimyrus isidori, Papyrocranus afer, Heterotis niloticus, Polypterus endlicheri et Schilbe mystus. Les raisons de changement de la composition spécifique d'un lac de barrage sont variables. Alhousseini (2002) note que les peuplements des lacs artificiels sont, à l'origine, ceux des cours d'eau qui les alimentent. Pour Lévêque (2005), la construction des barrages a pour conséquences, entre autres, la modification des peuplements des cours d'eau. Comme exemple, ces aménagements entrainent la disparition d'espèces rhéophiles en amont, compensée en partie par le développement d'espèces d'eau stagnantes et la disparition d'espèces qui se reproduisent dans les zones inondables à l'aval. Il faut cependant noter que la richesse spécifique de ce plan d'eau est proche des 38 espèces enregistrées dans le lac Ayamé 1 (Tah et al., 2009) et des 40 espèces dans celui d'Ayamé 2 (Adou et al., 2017) en Côte d'Ivoire. Elle reste cependant inférieure aux 74 espèces identifiées sur le cours principal du fleuve Bandama (Aboua et al., 2010), le lac de barrage Solomogou étant construit sur un des affluents de ce fleuve.

Les trois familles comptant le plus d'espèces sont d'abord les Cichlidae (10 espèces), suivis des Mormyridae (6 espèces) et des Cyprinidae (5 espèces). Cependant, l'abondance relative des familles dans les captures totales fait ressortir que les Cichlidae $(41,87 \%)$ suivis des Alestiidae $(14,47 \%)$ et des Mochokidae $(12,84 \%)$ dominent les captures. La forte représentativité des Cichlidae et des Mormyridae a également été signalée dans certains lacs de barrage en Côte d'Ivoire. En effet, Adou et al. (2017) ont rapporté que dans le lac d'Ayamé 2, les familles les plus riches en espèces sont les Cichlidae (11 espèces), suivis des Mormyridae ( 6 espèces) et des Alestidae ( 5 espèces). En outre, relativement au pourcentage numérique, les Cichlidae prédominent avec $35,04 \%$, suivis des Claroteidae et des Alestidae avec respectivement $28,89 \%$ et $24,91 \%$. Le même constat a été fait dans le lac Ayamé 1 où Tah et al. (2009) ont signalé une forte richesse spécifique chez les Cichlidae avec 9 espèces, suivis des Alestiidae et des Mormyridae avec respectivement 5 espèces chacune.

La représentativité des Mormyridae non moins négligeable en termes de nombre d'espèces pourrait traduire une bonne qualité de l'eau du lac de barrage. En effet, il est noté que ces poissons réagissent rapidement aux conditions d'altérations du milieu et seraient, par conséquent, de bons bio-indicateurs permettant de caractériser l'état présent d'un habitat (Niamien-Ebrottié et al., 2008; Byanikiro et al., 2017). Toutefois, leur nombre réduit dans les captures pourrait être imputé aux méthodes de capture. En tout état de cause, les engins et technique de pêche, ainsi que la sélectivité des engins de pêche pourraient influencer considérablement la structure des captures. 
Les valeurs de l'indice de diversité spécifique (H') de Shannon et Weaver et de l'indice d'équitabilité (E) de Piélou enregistrées sont respectivement 3.9159 et 0.7517. Elles sont plus grandes que celles calculées dans le lac de barrage Ayamé 2 où il a été enregistré $\mathrm{H}^{\prime}=2,32$ et $\mathrm{E}=0,63$. La valeur élevée de l'indice de diversité spécifique (H') de Shannon et le rapprochement de l'indice d'équitabilité (E) de Piélou un peu plus proche de 1 dans le lac de barrage Solomougou traduiraient une assez bonne organisation du peuplement ichtyologique dans cet hydrosystème, donc un état écologique beaucoup moins perturbé. En effet, une équitabilité proche de 1 traduit une bonne organisation du peuplement (Dajoz, 2000).

En outre, relativement aux périodes d'apparition des espèces dans les captures, les résultats indiquent que $60 \%$ d'entre elles ont des fréquences d'occurrence supérieures à $50 \%$. Elles sont alors constance dans les captures (Dajoz, 2000). Parmi les autres, quatorze espèces sont considérées comme accidentelles et une accessoire. Les variations saisonnière de certaines espèces ont également été signalées par différents auteurs dans différentes pêcheries (Ouédraogo et al., 2015).

\section{Conclusion}

Cette étude a permis de recenser trentesept espèces de poissons dans le lac de barrage Solomougou. Trois familles comptent le plus d'espèces. Ce sont les Cichlidae, les Mormyridae et des Cyprinidae. Cependant, en termes d'abondances relatives des familles, les Cichlidae $(41.87 \%)$ sont les plus abondants, suivis des Alestidae (14.47\%) et des Mochokidae (12.84\%). Enfin, quant aux abondances relatives des espèces, les trois espèces les plus représentatives sont Brycinus imberi $(14.41 \%)$, Synodontis schall $(12.76 \%)$ et Oreochromis niloticus (11.62\%). En outre, les différentes espèces montrent une certaine homogénéité dans leur distribution dans ce lac de barrage.

\section{CONFLIT D'INTERETS}

Les auteurs déclarent qu'il n'existe pas de conflit d'intérêts concernant cet article.

\section{CONTRIBUTIONS DES AUTEURS}

KDK et MD ont collecté les données sur le terrain. KDK, MD et YS ont participé à la rédaction de l'article. Enfin, $\mathrm{KN}$ a supervisé l'ensemble des travaux.

\section{REMERCIEMENTS}

Nous tenons à remercier le responsable de la Direction Régionale des Ressources Animales et Halieutiques du Département de Korhogo, qui a facilité nos rapports avec les pêcheurs. Nous remercions également les pêcheurs exerçant sur le lac de barrage Solomougou pour leur parfaite collaboration.

\section{RÉFÉRENCES}

Aboua RDB, Konan GN, Kouamelan PE, Berté S, Bamba M. 2010. Organisation spatiale du peuplement de poissons dans le Bandama. Int. J. Biol. Chem. Sci., 4(5): 1480-1493.

DOI: 10.4314/ijbcs.v4i5.65547

Adou YE, Blahoua KG, Bamba M, Yao SS, Kouamelan EP, N'Douba V. 2017. Premières données sur l'inventaire du peuplement ichtyologique d'un lac ouest Africain situé entre deux barrages hydroélectriques : Lac d'Ayamé 2 (Côte d'Ivoire). Journal of Applied Biosciences, 110:

10808-10818.

DOI: 10.4314/jab.v110i1.11.

Alhousseini S. 2002. Le poisson, une nouvelle ressource au barrage de Manantali. In Gestion Intégrée des Ressources Naturelles en Zones Inondables Tropicales, Colloques et Séminaires, Orange D, Arfi R, Kuper M, Morand P, Poncet Y (eds). Éditions IRD: Paris ; 501513.

Brou TY. 2005. Climat, mutations socioéconomiques et paysages en Côte d'Ivoire. Mémoire de synthèse des activités scientifiques présenté en vue de l'obtention de l'Habilitation à Diriger des Recherches, Université des Sciences et Techniques de Lille, France, p. 212.

Byanikiro RM, Nsila JN, Busanga AK, Ulyelali-Patho J, Micha J-C. 2017. Caractérisation et écologie des peuplements des Mormyridae dans la réserve de Yoko (Kisangani, RD Congo). 
Int. J. Biol. Chem. Sci., 11(3): 967-999. DOI : http://dx.doi.org/10.4314/ijbcs.v11i3.4

Cecchi P, Lévèque C, Aubertin C. 2007. L'Eau en Partage : les Petits Barrages de Côte d'Ivoire. Édition IRD : Paris ; 295p.

Da Costa KS, Tito de Morais L. 2007. Structures trophiques des peuplements de poissons dans les petits barrages. In L'Eau en Partage: Les Petits Barrages de Côte d'Ivoire, Cecchi P. Éditions IRD: Paris ; 153-164.

Da Costa KS, Tito de Morais L, Traoré K. 2004.Potentialités halieutiques des petits barrages du nord de la Côte d'Ivoire. Fiche technique, $7 \mathrm{p}$.

Dajoz R. 2000. Précis d'Ecologie. Dunod: Paris, France ; 615p.

FAO. 2018. La situation mondiale des pêches et de l'aquaculture. Résumé, FAO 2018, CA0191FR/1/07.18, 32p.

Koffi C. 2000. Aspects économiques de l'exploitation des ressources halieutiques des petits barrages du nord de la Côte d'Ivoire. Agronomie africaine, 12(1): 3349.

Le Guen T. 2002. Les barrages du Nord de la Côte-d'Ivoire: développement socioéconomique et état sanitaire des populations. Doctorat nouveau régime en Géographie, Université de Bretagne Occidentale, p. 467.

Lévêque C. 2005. Conséquences des barrages sur l'environnement. Les colloques de l'Académie d'Agriculture de France, irrigation et développement durable, 1: 33-34.

Liquete C, Cid N, Lanzanova D, Grizzetti B, Reynaud A. 2016. Perspectives on the link between ecosystem services and biodiversity: The assessment of the nursery function. Ecological Indicators, 63: 249-257. DOI: 10.1016/j.ecolind.2015.11.058.

Niamien-Ebrottié EJ, Konan KF, Gnagne T, Ouattara A, Ouattara M, Gourène G.
2008. Étude diagnostique de l'état de pollution du système fluvio-lagunaire Aby-Bia-Tanoé (Sus-Est, Côte d'Ivoire). Sud Sciences et Technologies, 16(1) : 513.

DOI : http://documents.irevues.inist.fr/bitstrea m/handle/2042/30379/sst16_2.

ORSTOM. 1962. Étude hydrologique du solomougou. Résultats de la Campagne 1961, 79p.

Ouédraogo R, Soara AE, Zerbo H. 2015. Caractérisation du peuplement piscicole du réservoir de Boalin, Ziniaré (Burkina Faso) deux décennies après l'introduction de Heterotis niloticus. Int. J. Biol. Chem. Sci., 9(5): 2488-2499. DOI : http://dx.doi.org/10.4314/ijbcs.v9i5.420

Piélou EC. 1966. The measurement of the diversity in different types of biological collections. J. Theor. Biol., 13: 131-144. DOI: 10.1016/0022-5193(66)90013-0.

Stiassny LJ, Teugels GG, Hopkins CD. 2008. Poissons d'Eaux Douces et Saumâtres de Basse Guinée, Ouest de l'Afrique Centrale. Édition IRD; 800p.

Shannon CE, Weaver W. 1963. The Mathematical Theory of Communication. University of Illinois Press; 117p.

Tah L, DA Costa KS, Kouassi NJ, Moreau J. 2009. Effort de pêche et production exploitée au lac d'Ayamé 1 (Bassin de la Bia ; Côte d'Ivoire) après le départ des pêcheurs « Bozos ». Agronomie Africaine, 21(1): 103-115.

Traoré K. 1996. État des connaissances sur les pêcheries continentales ivoiriennes. Rapport de consultation Avril 1996, PROJET FAO TCP / IVC / 4553.

Yapo RI, Mambo V, Alder AC, Ohou-Yao MJ, Ligban R, Dao D, Stamm C, Bonfoh B. 2016. Caractérisation saisonnière des eaux de puits à usage maraîchère et domestique de Korhogo (Côte d'Ivoire). Int. J. Biol. Chem. Sci., 10(3): 1433-1449. DOI: http://dx.doi.org/10.4314/ijbcs.v10i3.41 